\title{
Pulmonary Histiocytosis - Important to Recognize Pulmonary Hypertension as its Important Complication in Early Course of Disease
}

\section{Waqas Illyas* and Zohailb Tariq}

East Cheshire NHS Trust, Core Medical Trainee, United Kingdom

${ }^{*}$ Corresponding author: Ilyas W, East Cheshire NHS Trust, Core Medical Trainee, United Kingdom, Tel: +07711244097; Fax: +01604544566; Email: dr.waqas1000@hotmail.com

Rec date: March 10, 2016; Acc date: March 24, 2016; Pub date: March 30, 2016

Copyright: @ 2016 llyas W, et al. This is an open-access article distributed under the terms of the Creative Commons Attribution License, which permits unrestricted use, distribution, and reproduction in any medium, provided the original author and source are credited.

\section{Abstract}

A 45 year old male smoker presented with progressive shortness of breath for last 6 months. There was no significant past medical history. Physical examination and all blood tests were normal. Chest X-ray showed nonspecific interstitial infiltrates bilaterally.

High resolution CT scan was consistent with reticulonodular and cystic changes in upper and middle lobes and ground glass appearance in posterior basal lobes of lungs. There was mix obstructive and restrictive pattern on pulmonary function tests. Surgical Lung biopsy was taken which confirmed Pulmonary Langerhans cell histiocytosis. Patient was advised to stop smoking and started on medical management.

Unfortunately patient lost follow up and presented again with worsening symptoms and clinical signs of right sided heart failure. Echocardiography and right heart catheterization was performed which showed severe pulmonary hypertension. Patient was started on treatment for pulmonary hypertension which made little symptomatic improvement but his symptoms got worsen over time and is now being considered for lung transplantation.

Learning points/conclusion: Pulmonary function tests may be of normal, obstructive, restrictive or mixed pattern as is the case with our patient. But reduction in Diffusing capacity of Lung for Carbon Monoxide (DLCO) is the most common and earliest abnormality.

Pulmonary hypertension is a common and under recognized complication due to direct vasculopathy (arterioles but predominantly venules) and can occur at any time during course of disease as it may or may not corelate with lung function abnormalities and severity of parenchymal involvement. Investigations for pulmonary hypertension should be done early to prevent unnecessary delay in management.

Keywords: Pulmonary langerhans cell histiocytosis; Pulmonary hypertension; Langerhans cell histiocytosis;
Obstructive and restrictive lung disease; Rare cause of dyspnea; Reticulonodular; Cystic lung disease

\section{Introduction}

Pulmonary Langerhans Cell Histiocytosis (PLCH) is a relatively rare disease which presents with a common symptom of shortness of breath, its diagnosis is easily missed due to other common conditions causing similar clinical picture.

Main purpose of writing this case report is to highlight its differential diagnosis in young smokers who present with shortness of breath and also to remind some of the unique features of this disease on investigations and variable spectrum of its clinical presentation.

Pulmonary Hypertension is an important but often overlooked complication of PLCH, which can develop early in the course of disease. Its diagnosis is often delayed due to poor co relation with disease severity.

Interestingly a lot of studies have failed to demonstrate any relationship between severity of pulmonary hypertension and impairment of pulmonary function tests as it is primarily associated with pulmonary vasculopathy in contrast to other chronic lung diseases.

\section{Case Report}

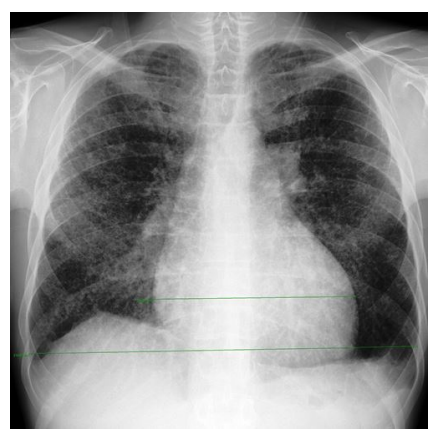

Figure 1: Diffuse reticular and cystic changes typically sparing costophrenic angles. 
A 45 year old male patient presented with gradual onset of shortness of breath for last 6 months. Other than smoking, there was no significant past medical or occupational history. Physical examination and blood tests were unremarkable. Initial Chest X-ray showed reticulo-nodular shadowing in both lung fields (Figure 1). He was advised to stop smoking and put on inhalers by his GP who then referred this patient to one of the respiratory physician for further workup and management.

\section{Investigations}

His High resolution CT scan chest showed multiple nodules and thick wall cysts of varying size and shapes (Honeycomb Pattern) bilaterally in upper and middle lobes while sparing posterior basal region. Basal regions showed centrilobular ground glass attenuation (Figure 2).

As a part of further workup Spirometry was performed that showed:

FEV1 $=2.37$ litre $(64 \%)$

$\mathrm{FVC}=3.71$ litre $(82 \%)$

FEV1/FVC $=64 \%$

TLC $=5.1$ (6.87 normal)

DLCO $=2.3(23 \%)$

$\mathrm{RV} / \mathrm{TLC}=34 \%(31 \%$ normal $)$
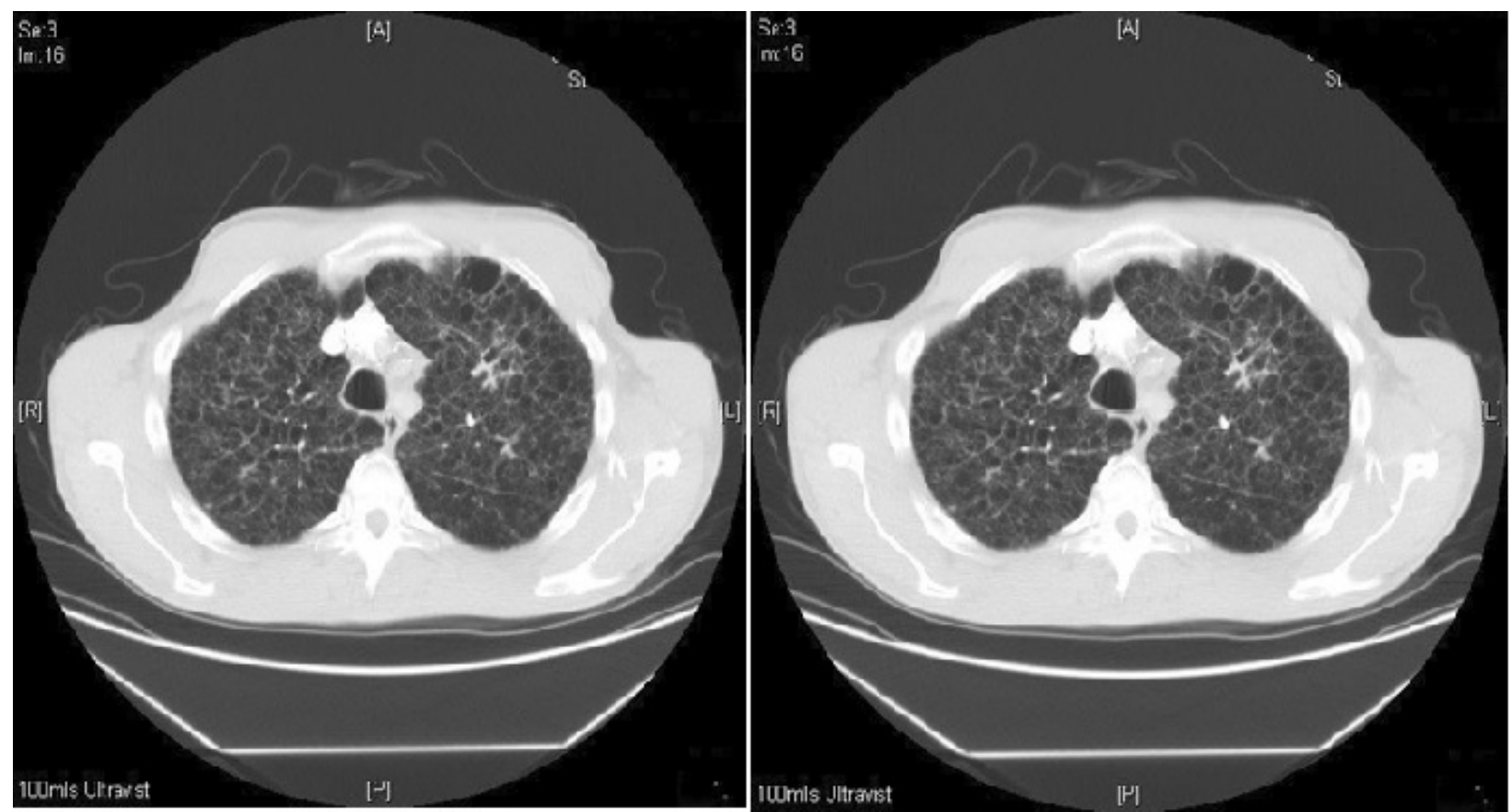

Figure 2: HRCT showing Gross Cystic Changes in upper and middle lung lobes.

Based unique radiological features and smoking history, diagnosis of Pulmonary Langerhans cell histiocytosis was strongly suspected which was confirmed latter on surgical lung biopsy results and further supported by positive immune cytochemistry for S100 and CD1a markers.

Patient was started on medical treatment and advised to quit smoking to slow the progression of disease. Unfortunately patient lost follow up for few years and presented again with worsening of shortness of breath. Physical examination this time showed clubbing, Loud P2 and raised jugular venous pulse. Repeated Pulmonary function tests were not significantly worse than previous ones. Keeping in view of possibility of pulmonary hypertension development, right heart catheterization and echocardiography were performed which showed Dilated Right ventricle and D-shaped LV cavity with mild systolic dysfunction.

His right heart catheterization results are as follows:

Right ventricular systolic pressure $=80 \mathrm{mmHg}$ (High)

\section{Pulmonary Artery Systolic Pressure: 78 mmHg (High)}

Mean Pulmonary Artery Pressure: 48 mmHg (High)

Treatment for pulmonary hypertension was initiated which made some improvement in patient's symptoms.

\section{Outcome}

Despite having optimal treatment for disease itself and pulmonary hypertension, patient symptoms continued to deteriorate to such an extent that patient now is being considered for Lung transplantation.

\section{Differential diagnosis}

Centrilobular Emphysema (most common): This disease generally tends to affect elder population and lacks nodularity on imaging. Young age group, combination of nodules and cysts typically in upper and middle lobes and smoking history are enough to think about PLCH. 
Lymphangiomyomatosis (LAM): Cysts of LAM are uniform rather round than irregular as in $\mathrm{PLCH}$ while predominance of cysts in upper and middle lobes again favors PLCH.

\section{Learning points}

Strongly suspect the possibility of PLCH in 20 to 40 years age group of smokers who have nodular and/or cystic lesions typically in upper and middle lung lobes.

Pulmonary function tests can be of normal, obstructive, restrictive or mixed pattern as is the case with our patient. But reduction in diffusing capacity of lung for carbon monoxide (DLCO) is the most common and earliest abnormality.

Pulmonary hypertension is a common but overlooked complication. It develops due to direct vasculopathy (arterioles but predominantly venules) and can occur at any time during course of disease as it may or may not co-relate with PFTs and severity of parenchymal involvement.

Investigations for pulmonary hypertension should be done early to prevent unnecessary delay in management.

\section{Discussion}

Pulmonary Langerhans cell histiocytosis (PLCH) is also called Histiocytosis $\mathrm{X}$ or Eosinophilic granuloma of lung. We are going to discuss 2 studies which show how Pulmonary Hypertension $(\mathrm{PH})$ as a complication of $\mathrm{PLCH}$ is associated with increased mortality and how can we improve quality of life by offering early treatment for it.
First study focused on 29 patients with $\mathrm{PLCH}-\mathrm{PH}$ having mean Pulmonary Arterial Hypertension (mPAH) of $45 \pm 14$ $\mathrm{mmHg}$ [1]. Out of these 12 patients were offered PH therapy which showed significant improvement in mPAH and mortality.

Second study demonstrated the dramatic response of PLCH related $\mathrm{PH}$ to vasodilator therapy. Patient was offered combination therapy of endothelin receptor antagonist, Bosentan and phosphodiesterase-5 inhibitor, Sildenafil. After 10 years, significant Echocardiographic and functional class improvement was seen [2].

\section{Conclusion}

Now with the advancement in science and research, we better understand the prevalence of $\mathrm{PH}$ in $\mathrm{PLCH}$ and increased mortality associated with it. Need of hour is to diagnose PLCH in young smokers who present with typical features and to pick up and treat associated $\mathrm{PH}$ early to reduce mortality and to improve quality of life.

\section{References}

1. Le Pavec J, Lorillon G, Jais X, Tcherakian C, Feuillet S, et al. (2012) Pulmonary Langerhans cell histiocytosis associated pulmonary hypertension: Clinical characteristics and impact of pulmonary arterial hypertension therapies. Chest 142: 1150-1157.

2. May A, Kane G, Yi E, Frantz R, Vassallo R (2015) Dramatic and sustained responsiveness of pulmonary Langerhans cell histiocytosis-associated pulmonary hypertension to vasodilator therapy. Respir Med Case Rep 14: 13-15. 